\title{
Intercalary frozen autograft for reconstruction of malignant bone and soft tissue tumours
}

\author{
Karem M. Zekry ${ }^{1,2}$ - Norio Yamamoto ${ }^{1}$ Katsuhiro Hayashi ${ }^{1} \cdot$ Akihiko Takeuchi $^{1}$ • \\ Takashi Higuchi $^{1}$ - Kensaku Abe ${ }^{1}$ - Yuta Taniguchi ${ }^{1}$ - Ali Zein A. A. Alkhooly ${ }^{2}$. \\ Ahmed Saleh Abd-Elfattah ${ }^{2}$ - Ezzat H. Fouly ${ }^{2}$. Adel Refaat Ahmed ${ }^{3}$. \\ Hiroyuki Tsuchiya ${ }^{1}$
}

Received: 26 November 2016/Accepted: 13 March 2017 / Published online: 25 March 2017

(C) The Author(s) 2017. This article is published with open access at Springerlink.com

\begin{abstract}
Purpose In 1999, we developed a technique using frozen autografts - tumour-containing bone treated with liquid nitrogen - for the reconstruction of malignant bone tumours. The purpose of this study was to evaluate the functional and oncological outcomes of frozen autografts for intercalary reconstruction of malignant bones and soft tissue tumours.

Methods This retrospective study was designed to assess 34 patients of mean age 35 (range, 6-79) years. The mean followup period was $62(24-214)$ months. The median length of the frozen autografts was $138.4 \pm 60.39(50-290) \mathrm{mm}$.

Results Postsurgically, 20 patients remained disease-free, seven patients survived with no evidence of disease, five patients were alive with disease, and two patients died of disease. The five- and ten-year survival rates of the frozen autografts were 91.2\% and the mean International Society of Limb Salvage score was $90 \%$. Complete bony union was achieved in $97 \%$ of the patients. There were five cases of nonunion, six cases of fracture, two cases of deep infection and four cases of local recurrence.

Conclusion Utilizing intercalary frozen autografts for patients with a nonosteolytic primary or secondary bone tumour without involvement of the subchondral bone is a good alternative
\end{abstract}

Karem M. Zekry

drkaremzekry@gmail.com

1 Department of Orthopaedic Surgery, Graduate School of Medical Science, Kanazawa University, Kanazawa, Japan

2 Department of Orthopaedic Surgery, Faculty of Medicine, Minia University, Minya, Egypt

3 Department of Orthopaedic Surgery, Faculty of Medicine, Alexandria University, Alexandria, Egypt treatment, because it is a straightforward biological technique and can provide excellent limb function.

Keywords Frozen autograft · Intercalary reconstruction · Malignant bone tumours

\section{Introduction}

Due to early diagnosis, effective chemotherapy, and precise preoperative imaging techniques, many tumours involving the metaphyseo-diaphyseal regions of long bones can be resected with joint preservation $[1,2]$. The intercalary tumour resection results in a segmental bone defect and can represent a challenging reconstructive problem. The surgical options for reconstructing these defects include biologic reconstructions such as an allograft [1-3], nonvascularised or vascularised fibular grafts [4, 5], autogenous extracorporeally-treated bone [6-10], bone transport [11, 12] or the combination of an allograft with vascularised fibular grafts. Nonbiologic reconstructions, on the other hand, use intercalary endoprostheses [13-15].

Biological reconstructions performed via reuse of the resected tumour-bearing bone have become increasingly popular; they include the use of extracorporeal irradiation [13], autoclaving [8], pasteurization [7] or freezing [6, 16]. A major advantage of these techniques is that the reconstructive procedure can be performed more easily when the dimensions of the defective excised bone match the grafted replacement bone [17]. Yamamoto et al. have described the use of freezing with liquid nitrogen at $-196{ }^{\circ} \mathrm{C}$ to treat the tumour-bearing bone; viable tumour cells are destroyed by inducing ice crystal formation and cell dehydration. Only one freeze cycle at $-196{ }^{\circ} \mathrm{C}$ for 20 minutes, followed by a thawing procedure, is required to kill tumour cells [16]. 
This retrospective study was performed to evaluate the clinical and oncological outcomes of patients who underwent intercalary frozen autograft reconstruction for malignant bone and soft tissue tumours of the extremities, and to observe the time required for bone integration and the incidence of any ensuing complications.

\section{Materials and methods}

Between April 1999 and July 2014, 50 patients with malignant bone and soft tissue tumours involving diaphyseal or metaphyseal regions of long bones underwent intercalary frozen autograft reconstructions (Table 1). All patients had adequate bone quality, and proximal and distal joints were preserved to maintain limb function. Of the 50 patients, 34 (68\%) were eligible for inclusion in this study because the following criteria were satisfied: (1) a minimum follow-up period of 24 months, (2) availability of complete imaging data and follow-up evaluation, (3) no previous treatment was received and (4) no pathological fracture. Cases were excluded from this study owing to short follow-up periods (out of 16 excluded cases, 14 were followed up for less than 24 months) or because follow-up data were not available ( 2 cases).

Fourteen males and 20 females were followed-up for a mean period of 62 months (range, 24-214). The mean age of the patients was 35 years (6-79). The pathological diagnoses were osteosarcoma ( 21 patients), metastatic disease ( 7 patients), and the remaining cases were rhabdomyosarcoma, leiomyosarcoma, undifferentiated pleomorphic sarcoma, chondrosarcoma, adamantinoma and phosphaturic mesenchymal tumour.

The tumours were located in the femur (18 patients), tibia (11 patients), humerus ( 3 patients) and radius ( 2 patients). The median length of the autografts was $138.4 \pm$ $60.39 \mathrm{~mm}$ (range, 50-290). A pre-operative work-up included a history, clinical evaluation, radiography, computed tomography (CT) of the lesion and chest, magnetic resonance imaging (MRI), technetium-99 $\mathrm{m}$ and thallium-201 bone scintigraphy and routine laboratory tests. Twenty-three patients received neoadjuvant and adjuvant chemotherapy. Another case with low-grade osteosarcoma received chemotherapy after local recurrence from surrounding residual soft tissue. Ethical approval for this study was granted from the Institutional Review Board of the Kanazawa University and written informed consent was obtained from all patients before surgical treatment including that the data may be included in future publications.

Wide resections were performed in 26 patients and intentional marginal resections were performed in eight patients who had a favourable response to chemotherapy; adjacent joints were preserved. In metaphyseal lesions, at least
$20 \mathrm{~mm}$ of tumour-free subchondral bone was essential for joint preservation. Per protocol, the tumour-bearing bone was snap frozen with liquid nitrogen and maintained at $-196{ }^{\circ} \mathrm{C}$ during a single 20 -minute treatment cycle, thawed at room temperature for 15 minutes and then rinsed with distilled water for ten to 15 minutes. There are two main types of freezing procedures. The first type is the Free-freezing Procedure (FFP) in which either two osteotomies or a hemicortical resection should be performed; thereafter, the tumour-bearing bone is totally immersed in liquid nitrogen after removal of the soft tissue component and curettage of the medullary canal (Fig. 1a). Hemicortical resections were performed if the preoperative imaging evaluation supported the rationale for an osteotomy around the lesion. FFPs were performed in 16 cases ( 2 osteotomies in 12 cases and a hemicortical resection in 4 cases). The second procedure is the Pedicle-Freezing Procedure (PFP) which requires a single osteotomy and the soft tissue to be divided along the extremity beyond the surgical margin until the arc of rotation is adequate. The surrounding soft tissue is protected by surgical sheets. The intramedullary canal is subsequently curetted to remove the bone marrow and contents of the tumour in order to prevent a graft-related fracture due to water expansion during freezing. The tumour-bearing bone from the limb being treated was rotated cautiously and frozen in liquid nitrogen while the surrounding soft tissue was continuously protected using surgical sheets. This pedicle-freezing method was performed under tourniquet control to reduce the risk of tumour dissemination and uncontrolled bleeding (Fig. 1b). PFP was performed in 18 cases. After freezing, fixation of the osteotomies was performed using intramedullary nails (4 cases), plates and screws (28 cases, including 1 plate [11 cases], 2 plates [ 14 cases], and 3 plates [ 3 cases]) and a combined nail and plate fixation ( 2 cases). In small osteolytic lesions, either a cement or bone graft may be used for mechanical support after curettage of the lesion.

Range of motion exercises for patients were started immediately after their operation. Partial weightbearing with the use of crutches was initiated two months post-operatively when appropriate. When a solid union was evident from a clinical and radiographic perspective, full weightbearing was allowed. Bone union was considered to be clinically evident when the patient did not experience pain while weightbearing, and radiographically evident when either the osteotomy line was no longer visible or there were bridging calluses of three cortices in the anteroposterior (AP) and lateral planes. Functional evaluations were performed using the International Society of Limb Salvage (ISOLS) scoring system [18].

The Kaplan-Meier method was used, with 95\% confidence interval, to estimate the survival rates for the patients and frozen autografts. Grafts were considered to have died if they had to be removed due to either graft-related or oncological complications. 


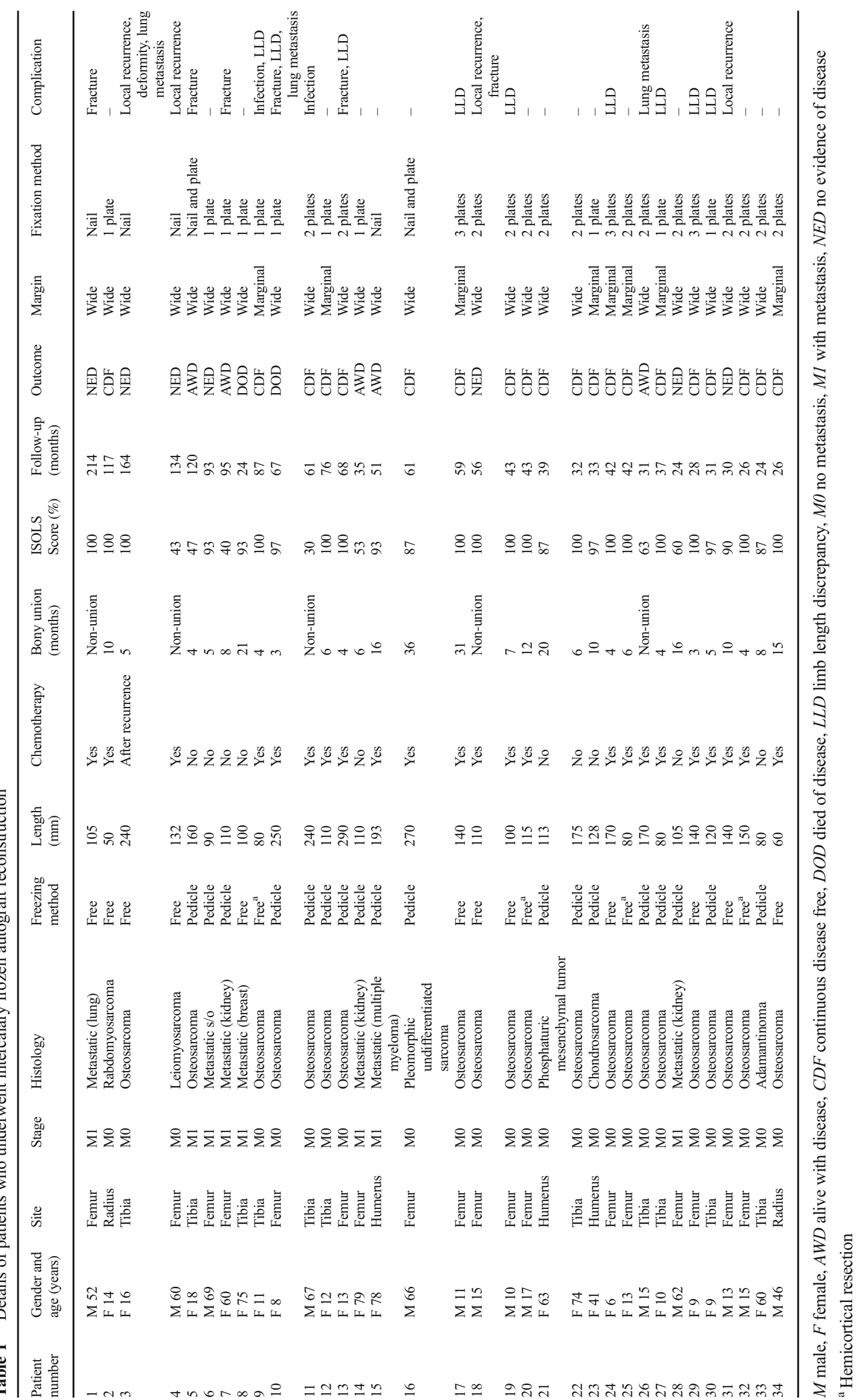


Fig. 1 Illustration showing (a) the free-freezing procedure in the distal femur and (b) pedicle freezing procedure in tibia a (1)

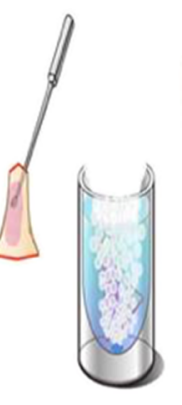

b

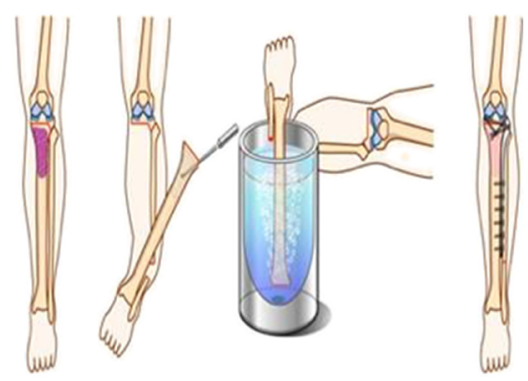

\section{Results}

At the last follow-up, 20 patients remained disease-free, seven patients had no evidence of disease, five patients were alive with disease, and two patients died of disease. During followup, the frozen autografts were removed in three cases (8.8\%); in two of those cases it was because of the local recurrence of disease from residual soft tissue around the femur, and in the other one case a severe infection developed in the tibia. Only one case with local recurrence underwent ablative surgery (hip disarticulation). The five- and ten-year survival rates of the patients were $97 \%$ and $94.1 \%$, respectively (Fig. 2). The five- and ten-year survival rates of the frozen autografts were $91.2 \%$ (Fig. 3), with survival rates of $94.4 \%$ and $87.5 \%$ for PFP and FFP autografts, respectively (Fig. 4). The mean ISOLS score for all patients was 26.1 points $(86.79 \%)$ and ranged between $30 \%$ and $100 \%$, and for the 31 patients who retained their frozen autografts the mean ISOLS score was 27 points (90\%) (Fig. 5).

Final bony union of the frozen autografts was achieved in 33 cases $(97 \%)$. The average bone union time was ten months

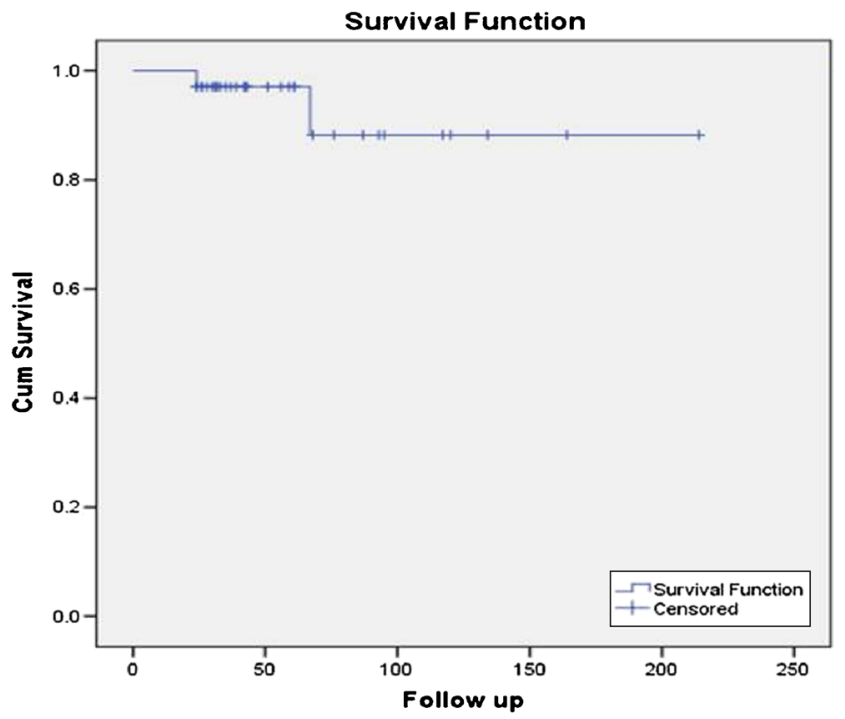

Fig. 2 Kaplan-Meier curve showing the patients five- and ten-year survival (range, 3-36) for the 29 patients who did not undergo additional surgery, 9.8 months for the 18 patients who received PFP autografts and 10.2 months for the 16 patients treated with FFP autografts. For the four patients who underwent a hemicortical resection, the mean time for bone union was 6.5 months and for those 12 patients who underwent two osteotomies, bone union took 11.7 months to achieve. There were five cases (14.7\%) of nonunion; among them, four cases were treated by bone graft augmentation at the nonunion site and one case required removal of the frozen autograft due to infection and a vascularised fibular graft was used instead.

Fractures occurred in six cases (17.6\%), all of which were managed by osteosynthesis with the addition of a bone graft in five cases. A deep infection occurred in two cases (5.9\%) at 5 and 44 months post-operatively. The infection in one case was managed by debridement and the plate was revised. In the other case, the frozen autograft was removed and replaced with a vascularised fibular graft.

Local disease recurrence from surrounding soft tissue occurred in four cases (11.8\%), all of them after the FFP. In three cases, the locally recurring malignancy was excised, but it

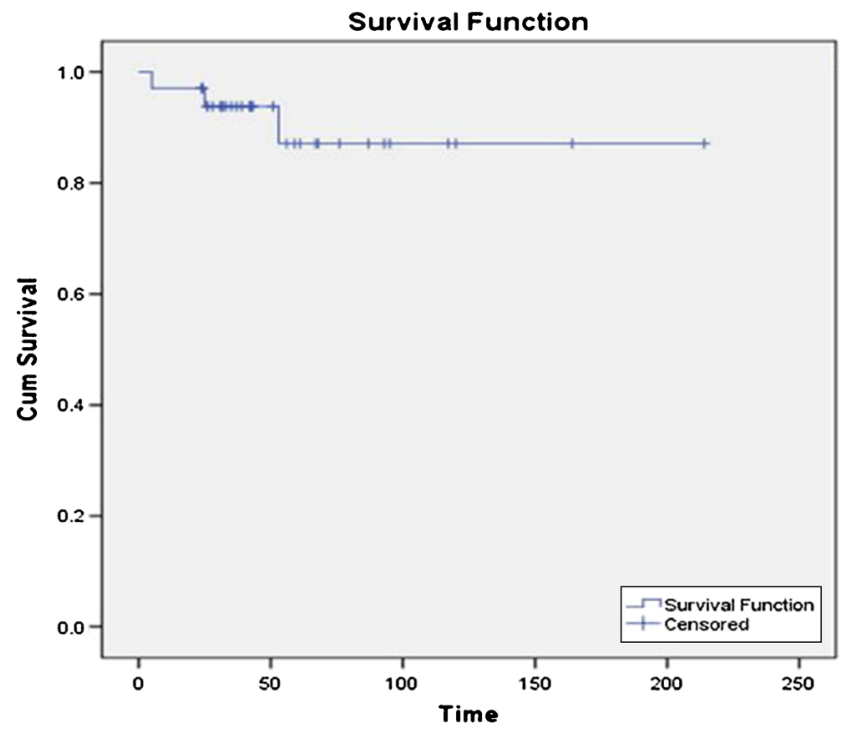

Fig. 3 Kaplan-Meier curve showing the graft five- and ten-year survival 


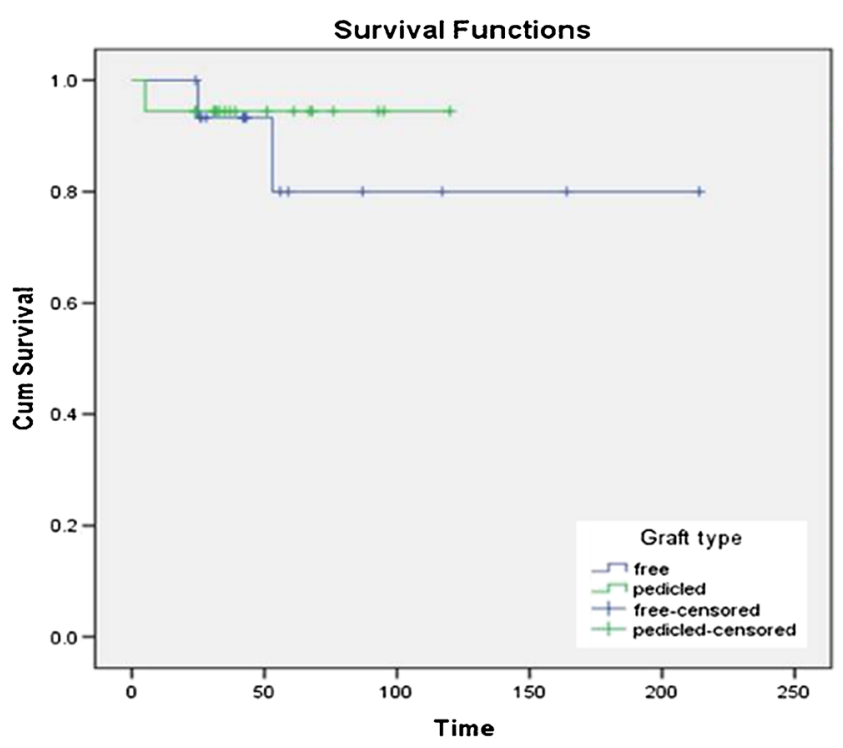

Fig. 4 Kaplan-Meier curve showing graft survival according to the type of reconstruction

recurred again in one case and had to be treated with hip disarticulation. In another case, malignant cells recurred very close to the bone, invading the distal femur and was managed by conversion to a tumour prosthesis, removal of the frozen autograft and only the junctional area between the host and frozen bone could be preserved. No disease had recurred within the frozen autograft. During follow-up, three patients that had osteosarcoma developed lung metastases; thoracoscopic a

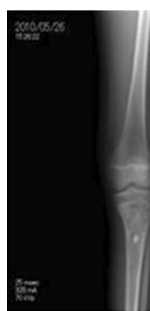

d

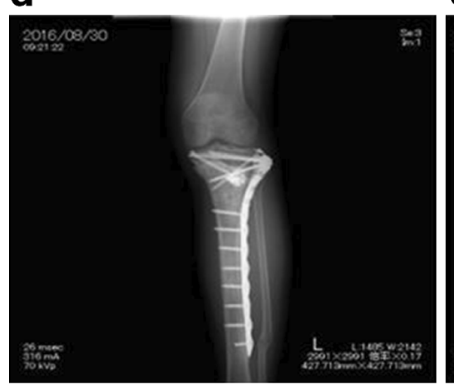

e

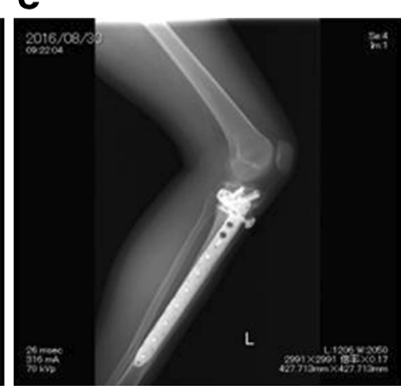

Fig. 5 Case presentation representing a 12-year-old girl with osteosarcoma of the left proximal tibia at the time of her surgery. a Anteroposterior radiograph of a proximal tibia showing the tumour. b T1-weighted MRI of the proximal tibia showing the tumour mass with low signal intensity. $\mathbf{c}$ Intraoperative radiograph showing the pedicled and resected segment to be frozen and the host bone. d, e Radiographs of the proximal tibia at the time of final follow-up excision and/or open thoracic excision of the metastases or the lung segment(s) was performed by thoracic surgeons.

During follow-up, a limb deformity was observed in one case; it was corrected by the application of a Taylor spatial frame (TSF). Leg-length discrepancies (LLDs) occurred in nine patients. The average LLD at the last follow-up was $31 \mathrm{~mm}$ (range, 16-50). The LLD was due to the destruction of the physis, either because of tumoural invasion or scarification during surgical resection and fixation. LLD was corrected in three cases via a limb lengthening procedure using a TSF which was performed in virgin bone. If the frozen autograft reconstruction was done in the femur, lengthening of the tibia was performed, and vice versa. Other cases were managed conservatively using shoe lifts and by monitoring progress.

\section{Discussion}

Although many malignant bone tumours are close to or involve the subchondral bone, diaphyseal and meta-diaphyseal involvement is not infrequent for primary as well as metastatic lesions. If preservation of an adjacent joint can be achieved, functional outcomes are typically superior [19].

There are many options for reconstruction after the intercalary resection of malignant bone tumours in extremities. An intercalary prosthesis is an option to provide immediate stability and early weightbearing capability, but the risk of complications, such as loosening, infection, implant breakage and fracture of the adjacent bone may be higher than those for biological reconstructions [14]. Ruggieri et al. [15] reported that $29 \%$ of patients developed mechanical complications, including loosening, rotational instability, breakage and disassembly of prostheses that mostly occurred in reconstructions requiring more than $10 \mathrm{~cm}$ of bone to be resected; therefore, it was concluded that in defects exceeding $10 \mathrm{~cm}$ in length the indication for intercalary prosthesis should be reconsidered.

Allografts have been widely used for intercalary reconstruction of malignant bone tumours; however, allografts require a bone-banking system. Allograft reconstruction has the potential for many complications such as nonunions, fractures, infection with possibility of autoimmune responses and infectious disease transmission [20,21]. Bus et al. reported that complications occurred in $76 \%$ of patients and reoperations were necessary in $70 \%$. The nonunion, fracture and infection rates were $40 \%, 29 \%$ and $14 \%$, respectively, and $17 \%$ of allografts were removed. The complication rate was so high that the authors of this report suggested that the use of allografts for reconstruction of defects larger than $15 \mathrm{~cm}$ should be reconsidered, especially in older patients [3].

Nonvascularised and vascularised fibular autografts are available options for intercalary reconstruction; however, they have complications associated with donor site morbidity [13]. 
A nonvascularised fibular graft in the absence of biological activity runs the risk of resorption and nonunion [4, 9]. Vascularised fibular grafting does not have the benefit of a sufficiently thick original bone, thus delaying full weightbearing until several years after the surgery, and the patients need crutches for extended periods. Furthermore, stress fractures, nonunions, malunions and infections represent other possible complications [11].

Combining an allograft with a vascularised fibula graft was introduced to overcome the complications associated with an allograft alone, and the combined procedure achieves better results, although anastomoses are demanding and the procedure is time-consuming and not devoid of complications at the donor site [10].

Bone transport is another alternative procedure, but it requires patient co-operation and is associated with complications such as pin tract infections, reduced range of motion, delayed consolidation and union problems at the docking site, and malalignment after the distraction period, especially in patients with large bone defects [12].

Several techniques have been developed to recycle the resected bone, including autoclaving, irradiation and pasteurization, but these methods demand strict thermal control and special equipment [6]; furthermore, heat treatment by autoclaving technique results in a weakening of the integrity of bones and a loss of the ability for bone induction [6, 22]. Autoclaved or irradiated autografts have been associated with a high rate of complications such as nonunion, fracture and infection [23].

Khattak et al. reported a mean bone union time of 24.2 months and an infection rate of $41.6 \%$ in his reconstruction study that used tumour-bearing bone that had been autoclaved [8]. In a study reported by Krieg et al., the nonunion rate was $16 \%$ using extracorporeal irradiated autografts [9]. In another study investigating pasteurised autografts, Manabe et al. reported a $20 \%$ infection rate and mean bone union time of 12 months [7]. Qu et al. reported an 11.1\% infection rate and a mean bone union time of 11 months in a study of intercalary reconstructions with devitalised tumourbearing bone treated in a $20 \%$ sterile saline solution at $65{ }^{\circ} \mathrm{C}$ for 30 minutes [10].

The efficacy of treatment with liquid nitrogen on osteosarcoma cells has been documented by Yamamoto et al., both in vitro and in vivo; also, frozen autografts were found to maintain adequate biomechanical properties [16]. Takata et al. reported that bone morphogenetic activity was better preserved in frozen autografts treated with liquid nitrogen than in those treated by autoclaving or pasteurization [24].

Intercalary reconstruction with the use of a frozen autograft has many advantages; it is a relatively simple and low cost technique, allows a precise fit between the graft and host bone, preserves osteoinduction and osteoconduction [24], averts graft rejection, avoids the transmission of infectious diseases, preserves the bone stock and biomechanical strength, allows easy attachment of ligaments and tendons to bone, prevents harmful denaturation of substances and provides early revitalization with possible cryoimmunological effects [17].

The five- and ten-year survival rates of frozen autografts were $91.2 \%$, with survival rates of $94.4 \%$ and $87.5 \%$ for pedicled- and free-frozen autografts, respectively, which is similar to or better than current reports. In a study of intercalary reconstructions with devitalised tumour-bearing bone treated in a $20 \%$ sterile saline at $65^{\circ} \mathrm{C}$ for 30 minutes, Qu et al. reported a five-year survival rate of $86.4 \%$ [10]. Manabe et al. [7] reported an $80 \%$ graft survival rate for pasteurised autografts. In their studies of allograft reconstructions, AponteTinao et al. [2] and Muscolo et al. [1] reported graft survivals of $76 \%$ and $79 \%$, respectively.

In our study, final bony union of the frozen autografts was achieved in 33 cases (97\%). The average bone union time for the 29 patients who did not require additional surgery was ten months (range, 3-36) and the time was not significantly different between the FFP (10.2 months) and the PFP ( 9.8 months). In a previous study performed by Shimozaki et al., bone union was reportedly 9.8 months using FFP and 4.8 months using the PFP [25]. In our study, the nonsignificant difference between procedures could be explained by the older mean age of patients treated with the PFP (45 years) than with the FFP (24 years); moreover, the FFP also included the hemicortical resection group which had a shorter mean bone union time (6.5 months). There were five (14.7\%) cases of nonunion in this study. In four cases, the nonunion was treated by a bone graft augmentation at the nonunion site, and in one case the nonunion was due to an infection requiring the frozen autograft to be removed and converted to a vascularised fibular graft. Fractures occurred in six cases (17.6\%), but all of these cases were managed by osteosynthesis with a supplementary bone graft in five cases.

In this study, infection occurred in two cases (5.9\%) and local recurrence from surrounding soft tissue occurred in four cases $(11.8 \%)$. Only one of these cases ended with ablation of the extremity. No local recurrence was observed within a frozen autograft.

Evaluation of LLD during the follow-up of pediatric patients should be considered because it is an unavoidable late sequela if the physis is invaded by the tumour or sacrificed during surgical resection. Bone lengthening is usually considered if the LLD is more than $2 \mathrm{~cm}$ and the procedure is performed in virgin bone. If the frozen autograft reconstruction was done in the femur, lengthening of the tibia was performed, and vice versa. In our study, lengthening procedures were performed in three patients using a TSF.

Despite reports from Yamamoto et al. indicating that frozen bone has sufficient biomechanical strength for limb reconstruction, the presence of a serious osteolytic lesion is a contraindication for the freezing technique [16]. If the osteolytic 
lesion is small, the freezing technique may be possible, albeit with curettage and a bone graft or cementation of the lesion, provided that the internal fixation is secure.

Utilizing intercalary frozen autografts for patients with nonosteolytic primary or secondary bone tumours without involvement of the subchondral bone is a good alternative treatment, because it is a straightforward biological technique and can provide excellent limb function.

A limitation of this study is that it presents level IV evidence from a retrospective study. The patients included in this study had different diagnoses, tumour locations, and fixation methods, all of which made it difficult to analyze the differences in complications. The number of patients was small because many patients with metaphyseal lesions were treated with prosthetic replacement or a frozen autograft prosthetic composite in the first years of the study period.

Acknowledgements The authors acknowledge Mr. T. Nomura for preparation of the illustrations.

\section{Compliance with ethical standards}

Conflict of interest The authors declare that they have no conflicts of interest.

Funding There is no funding source to declare.

Ethical approval The study received ethical approval from the Institutional Review Board of the Kanazawa University, and written informed consent was obtained from all patients before surgical treatment including that the data may be included in future publications.

Open Access This article is distributed under the terms of the Creative Commons Attribution 4.0 International License (http:// creativecommons.org/licenses/by/4.0/), which permits unrestricted use, distribution, and reproduction in any medium, provided you give appropriate credit to the original author(s) and the source, provide a link to the Creative Commons license, and indicate if changes were made.

\section{References}

1. Muscolo DL, Ayerza MA, Aponte-Tinao L, Ranalletta M, Abalo E (2004) Intercalary femur and tibia segmental allografts provide an acceptable alternative in reconstructing tumor resections. Clin Orthop Relat Res 426:97-102

2. Aponte-Tinao L, Farfalli GL, Ritacco LE, Ayerza MA, Muscolo DL (2012) Intercalary femur allografts are an acceptable alternative after tumor resection. Clin Orthop Relat Res 470:728-734

3. Bus MP, Dijkstra PD, van de Sande MA et al (2014) Intercalary allograft reconstructions following resection of primary bone tumors: a nationwide multicenter study. J Bone Joint Surg Am 96:e26

4. Chen Z, Chen Z, Zhang G (1997) Fibula grafting for treatment of aggressive benign bone tumor and malignant bone tumor of extremities. Chin Med J (Engl) 110(2):125-128

5. Chen CM, Disa JJ, Lee HY et al (2007) Reconstruction of extremity long bone defects after sarcoma resection with vascularized fibula flaps: a 10-year review. Plast Reconstr Surg 119:915-924
6. Tsuchiya H, Wan SL, Sakayama K, Yamamoto N, Nishida H, Tomita K (2005) Reconstruction using an autograft containing tumour treated by liquid nitrogen. J Bone Joint Surg (Br) 87:218-225

7. Manabe J, Ahmed AR, Kawaguchi N, Matsumoto S, Kuroda H (2004) Pasteurized autologous bone graft in surgery for bone and soft tissue sarcoma. Clin Orthop Relat Res 419:258-266

8. Khattak MJ, Haroon-ur-Rasheed UM, Umar M (2006) Autoclaved tumor bone for reconstruction: an alternative in developing countries. Clin Orthop Relat Res 447:138-144

9. Krieg AH, Davidson AW, Stalley PD (2007) Intercalary femoral reconstruction with extracorporeal irradiated autogenous bone graft in limb-salvage surgery. J Bone Joint Surg (Br) 89:366-371

10. Qu H, Guo W, Yang R et al (2015) Reconstruction of segmental bone defect of long bones after tumor resection by devitalized tumor-bearing bone. World J Surg Oncol 13:282

11. Tsuchiya H, Tomita K, Minematsu K et al (1997) Limb salvage using distraction osteogenesis: a classification of the technique. $\mathrm{J}$ Bone Joint Surg (Br) 79:403-411

12. Demiralp B, Ege T, Kose O, Yurttas Y, Basbozkurt M (2014) Reconstruction of intercalary bone defects following bone tumor resection with segmental bone transport using an Ilizarov circular external fixator. J Orthop Sci 19:1004-1011

13. Ahlmann ER, Menendez LR (2006) Intercalary endoprosthetic reconstruction for diaphyseal bone tumours. J Bone Joint Surg (Br) 88:1487-1491

14. Hanna SA, Sewell MD, Aston WJ et al (2010) Femoral diaphyseal endoprosthetic reconstruction after segmental resection of primary bone tumours. J Bone Joint Surg (Br) 92:867-874

15. Ruggieri $P$, Mavrogenis AF, Bianchi G, Sakellariou VI, Mercuri M, Papagelopoulos PJ (2011) Outcome of the intramedullary diaphyseal segmental defect fixation system for bone tumors. J Surg Oncol 104:83-90

16. Yamamoto N, Tsuchiya H, Tomita K (2003) Effects of liquid nitrogen treatment on the proliferation of osteosarcoma and the biomechanical properties of normal bone. J Orthop Sci 8:374-380

17. Nishida J, Shimamura T (2008) Methods of reconstruction for bone defect after tumor excision: a review of alternatives. Med Sci Monit 14:RA107-RA113

18. Enneking WF, Dunham W, Gebhardt MC, Malawar M, Pritchard DJ (1993) A system for the functional evaluation of reconstructive procedures after surgical treatment of tumors of the musculoskeletal system. Clin Orthop Relat Res 286:241-246

19. Fuchs B, Ossendorf C, Leerapun T, Sim FH (2008) Intercalary segmental reconstruction after bone tumor resection. Eur J Surg Oncol 34:1271-1276

20. Sugiura H, Takahashi M, Nakanishi K, Nishida Y, Kamei Y (2007) Pasteurized intercalary autogenous bone graft combined with vascularized fibula. Clin Orthop Relat Res 456:196-202

21. Campanacci DA, Puccini S, Caff G et al (2014) Vascularised fibular grafts as a salvage procedure in failed intercalary reconstructions after bone tumour resection of the femur. Injury 45:399-404

22. Urist MR, Dawson E (1981) Intertransverse process fusion with the aid of chemosterilized autolyzed antigen-extracted allogeneic (AAA) bone. Clin Orthop Relat Res 154:97-113

23. Chen TH, Chen WM, Huang CK (2005) Reconstruction after intercalary resection of malignant bone tumours: comparison between segmental allograft and extracorporeally-irradiated autograft. J Bone Joint Surg (Br) 87:704-709

24. Takata M, Sugimoto N, Yamamoto N et al (2011) (2011) Activity of bone morphogenetic protein-7 after treatment at various temperatures: freezing vs. pasteurization vs. allograft. Cryobiology 63:235239

25. Shimozaki S, Yamamoto N, Shirai T et al (2014) Pedicle versus free frozen autograft for reconstruction in malignant bone and soft tissue tumors of the lower extremities. J Orthop Sci 19:156-163 